of Redondo Beach, California and Ball Aerospace of Boulder, Colorado borrows from research on large space mirrors developed for military satellites. The segmented primary mirror divides into three panels, which, once in space, will unfold like flower petals. The technical difficulty of testing this system is the main reason for a slip in the launch date from 2008 to 2010.

Once in space, the mirror, which will be made of glass or beryllium, will be tested and adjusted every few weeks to compensate for any changes in shape caused, for example, by heating.

These and other new technologies have pushed up costs, however. Last year, astronomers reluctantly agreed to NASA's proposal to decrease the size of the mirror from 8 to 6 metres, reducing the telescope's resolving power by $25 \%$ and its light-collecting ability by $44 \%$. An orbital test of a scaled-down mirror was also scrapped.

Some outside observers worry that the current budget is still too small, but Seery says his team is far more savvy about the technological challenges and true costs than it was.

Others say that it is right to push the state of the art forward with the planned new telescope.

"These kinds of telescopes only come along once every decade or so," says Jonathan Lunine, a planetary scientist at the University of Arizona in Tucson and a member of the Webb's recently appointed Science Working Group, which will meet for the first time next week. "Because of the size and the scope of this mission, the opportunity to do new technology is really there."

http://ngst.gsfc.nasa.gov

\title{
Prion research stepped up as fear grows of deer disease
}

\section{Rex Dalton and Erika Check}

As concern mounts in the western United States about a relative of mad cow disease found among deer and elk, federal agencies are boosting research into this family of fatal neurodegenerative conditions.

Awareness of chronic wasting disease (CWD) has risen in the past few years. The disease, present in the Rocky Mountain states since the 1960s, has recently been found in the midwest (see Nature 416, 569-570; 2002). Officials are also concerned that mad cow disease might enter the United States.

CWD, mad-cow disease and the human form, Creutzfeldt-Jakob disease (CJD), are all thought to be caused by infectious prions - misshapen forms of a protein that can convert the normal version to their own rogue shape.

There is no evidence that CWD has caused human disease, but this remains a possibility as $60 \%$ of people in the affected region eat venison or elk. Fears about CWD have been fuelled by reports of venisoneaters who have died with neurological symptoms, although none was found to have had a prion disease.

The Department of Defense is leading the push for more research with its National Prion Research Program. The Institute of Medicine held a meeting in Washington on 12-13 September to advise on priorities for the first grants, worth $\$ 42.5$ million.

High on the agenda was the development of better diagnostic tests that could reveal infection before symptoms emerge. Cases of prion disease are currently confirmed

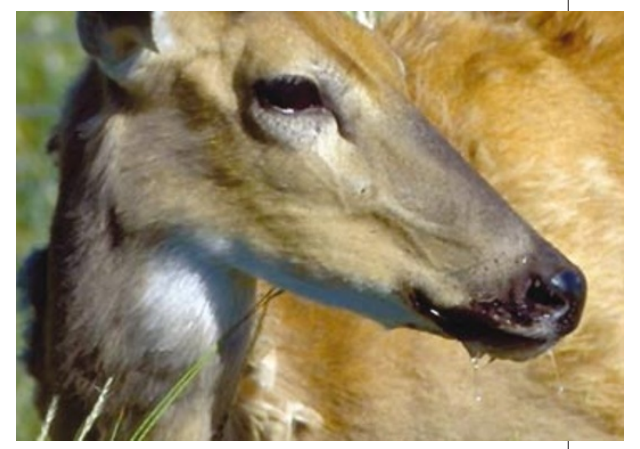

Waste concern: can disease pass to humans from animals such as this infected white-tailed deer?

by taking biopsies of tonsils or brain tissue.

The Department of Defense also wants to award grants to increase the size of the small US prion-research community. Researchers are put off by biosafety requirements and may be fearful of working with prions, says Pierluigi Gambetti of Case Western Reserve University in Cleveland, Ohio. "The reality is that you have to pay people more to work in your laboratories," he says.

Gambetti heads the National Prion Disease Pathology Surveillance Center, which examines cases of neurological disease and maintains CJD tissue samples. Its budget is to rise fourfold to $\$ 1$ million next year.

In addition, the National Institutes of Health wants to create a repository for reagents used in prion research. It is also considering creating a central repository for antibodies, which are necessary for the development of diagnostic tests.

\section{Japan celebrates safe launch after string of problems}

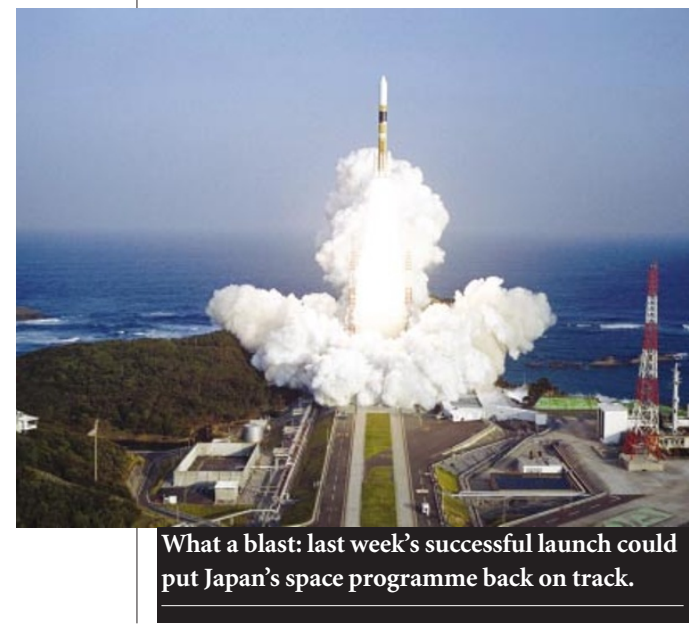

David Cyranoski, Tokyo

The successful launch of Japan's H-IIA rocket last week provided a welcome boost to the country's troubled rocket programme, before a crucial international launch later this year.

On 10 September, the National Space Development Agency of Japan (NASDA) used H-IIA to launch satellites designed to conduct data-relay and zero-gravity research. The H-IIA series and its predecessor, the $\mathrm{H}$-II, have had several failures. The most recent occurred in February, when the rocket failed to release a $¥ 600$-million (US\$4.9-million) satellite designed to measure the impact of high temperatures during re-entry.

The next H-IIA launch, scheduled for later this year, will be a major test. It will carry the Advanced Earth Observing Satellite II, with detectors made by NASDA, NASA and the CNES, France's national space agency. They will monitor chlorophyll, water vapour, sea surface temperature, sea ice and ocean wind velocity.

The mission will also include Australia's first scientific satellite launch for 30 years. FedSat, designed by Australia's Cooperative Research Centre for Satellite Systems, will carry out engineering, communication and science experiments.

NASDA hopes that more successes will help to establish Japan's space programme as commercially viable. It plans to turn over operations of the H-IIA to Mitsubishi Heavy Industries in the next two years. 